AUTHOR'S POST PRINT (Romeo Colour: Green)

Acta Astronautica (ISSN: 0094-5765) Vol. 48, Nos. 5-12, pp. 603-615, 2001.

DOI: $10.1016 / \mathrm{S} 0094-5765(01) 00031-5$

Publisher version available at

http://www.sciencedirect.com/science/article/pii/S0094576501000315

\title{
ON THE CONVECTIVE DISTURBANCES INDUCED BY G-JITTER ON THE SPACE STATION
}

\author{
R. MONTI, R. SAVINO, M. LAPPA* \\ Università degli Studi di Napoli "Federico II" \\ Dipartimento di Scienza e Ingegneria dello Spazio "Luigi G. Napolitano" \\ P.le V.Tecchio 80, 80125 Napoli (Italy) \\ *current e-mail address: marcello.lappa@strath.ac.uk
}

\begin{abstract}
A numerical tool has been prepared to quickly predict the overall disturbances for the typical Microgravity Environment (MGE) on the ISS. A reference study case (that shows a large sensitivity to acceleration disturbances) is identified and numerical simulations are carried out to compare the results of the solution of the full Navier-Stokes equations with a time-dependent acceleration (that give the instantaneous time-dependent flow) with the solutions of the time-averaged field equations (Gershuni formulation), containing all the g-jitter terms (like those that identify the microgravity environment of the ISS) grouped in a single parameter. The paper shows that the overall disturbances of the thermo-fluid-dynamic field are easily evaluated assigning as input to the fluiddynamic code a single (equivalent) frequency g-jitter (in the direct formulation) or an overall vibrational Rayleigh number (in the time-averaged formulation). The code is validated and applied to the problem of thermodiffusion in a typical metal alloy.
\end{abstract}

\section{$\underline{1 . \text { Introduction }}$}

Previous theoretical and numerical studies carried out at the University of Naples ${ }^{1}$ have addressed the problem of the thermo-fluiddynamic response of Fluid Physics experiments to relatively high frequency gjitter. One of the key results of the studies is that, at sufficiently high frequencies, the velocity oscillates around a non-zero average value with the g-jitter period and the scalar quantities (temperature and/or species distributions) are similarly made up by a steady plus an oscillatory contribution.
For the typical g-jitter prevailing in a large part of the frequency spectrum of the ISS (e.g. for frequencies $\mathrm{f}>0.1 \mathrm{~Hz}$ ), the amplitudes of the oscillation of the temperature (or concentration) distortions are negligible compared to the steady, time-averaged distortions, arising from thermovibrational effects (associated to the non-zero average part of the velocity field).

This fact proves to be of a fundamental advantage for the evaluation of the convective effects induced by g-jitter since the oscillatory part of the disturbances can be neglected, compared to the time-averaged disturbances, and the time-averaged fields can be simply computed (i.e. with much less computation time) by solving only the time-averaged form of the field equations (Gershuni formulation ${ }^{2}$ ).

Different study cases (that show a large sensitivity to vibration accelerations) have been identified and numerical simulations have been carried out to compare the results of the full Navier-Stokes equations with a time-dependent periodic body force (that give the instantaneous time-dependent flow) with the solutions of the time-averaged field equations, containing all the g-jitter terms (like those that identify the microgravity environment of the ISS) grouped in a single parameter.

In this paper a number of examples are worked out for a reference study case and for a typical case very often proposed for microgravity experimentation (thermodiffusion).

2. Thermo-fluid-dynamic disturbances in a microgravity environment

The goal of all the microgravity experimentations is the obtainment of a 
Product (be it a material, a measurement of a thermophysical property, a protein, etc.); one expects that this Product (symbolically denoted by P) will somehow be "better" that those achievable on ground at $1-\mathrm{g}\left(\mathrm{P}_{1}\right)$. A Microgravity Relevance factor (MR) can be defined just in terms of the difference of these two Products $\left(\Delta \mathrm{P}_{1}=\mathrm{P}_{1}-\mathrm{P}\right)$ : only if this difference is large enough (in an appropriate scale) one can claim that the experiment is worth doing in microgravity (instead at the 1g).

If a 0 -g reference state is taken one can similarly define another symbolic difference $\Delta \mathrm{P}_{0}=\mathrm{P}-\mathrm{P}_{0}$ between the product that can be achieved in the real microgravity environment (P) and the product $\left(\mathrm{P}_{0}\right)$ that could be (ideally) achieved in a 0 -g environment; this difference is typically due to the existing non $0-\mathrm{g}$ Microgravity Environment (MGE). A suitable $M G$ platform for a given class of experiment is one that guarantees a small value of $\Delta \mathrm{P}_{0}$. Most significant microgravity experiments are those that exhibit large "values" of $\Delta \mathrm{P}_{1}$, and small values of $\Delta \mathrm{P}_{0}$.

In most of the cases Fluid Physics experiments and Material Science processes lead to a product $(\mathrm{P})$ that is strongly related to the thermofluidynamic field. For instance in 0 -g a thermofluidynamic field (TFD) is established in fluid phases (typically quiescent, or quasi quiescent conditions, i.e. purely diffusive processes) leading to the product $\mathrm{P}_{0}$.

In presence of accelerations a different TFD field is established; if these accelerations are "small" the TFD can be thought to consist of the previous $\mathrm{TFD}_{0}$ plus a relatively "small" disturbance.

Having defined the disturbance $\Delta \mathrm{P}_{0}$ and assumed the dependence P(TFD), within a small range of accelerations one can always linearize and get $\Delta \mathrm{P}_{0}=\mathrm{P}-\mathrm{P}_{0}=\mathrm{K}\left(\mathrm{TFD}-\mathrm{TFD}_{0}\right)$ and relate the overall Product difference to the thermofluidynamic fields disturbances. For the selected cases (0-g, purely diffusive, quiescent reference state) the only difference in the TFD is caused by the convective velocity field induced by the prevailing accelerations $\underline{V}(\underline{g})$.
Most of the time it is the ratio between the convective term over the diffusive one in the scalar transport equations that gives a measure of the TFD disturbance. The order of magnitude of this ratio is measured by the Peclet number $\left(\mathrm{Pe}=\mathrm{V} / \mathrm{V}_{\mathrm{d}}\right)$ where $\mathrm{V}_{\mathrm{d}}$ is the diffusive velocity.

In conclusion the evaluation of the order of magnitude of the disturbance can be done by an appropriate evaluation of the order of magnitude of the convective velocity that in turn depends on the accelerations prevailing on the microgravity platform.

In what follows we make the following assumptions:

a) The acceleration field is responsible for the generation of a velocity field;

b) This velocity field alters the diffusive transport and the distribution of scalar quantities;

c) The different distribution of these scalar quantities give rise to products $(\mathrm{P})$ that are different from the 0 -g product $\left(\mathrm{P}_{0}\right)$.

The problem of the suitability of a microgravity platform is therefore reduced to the computation of the time and space distribution of a number of parameters (say temperature and/or concentrations) at 0 -g and at the MGE and in evaluating their difference at some points and times. This approach may be misleading if one makes an arbitrary selection of $\underline{r}$ and $t$. For instance at the boundaries, where parameters are imposed in both cases; $\Delta_{0} T$ and $\Delta_{0} c$ are both zero; similarly if one chooses time $\mathrm{t}=0$ (or, in some other case, $t \rightarrow \infty$ ).

It is also obvious that dimensional quantities, say $\Delta_{0} T(\underline{r})$, cannot give any idea of the importance of these differences: only when these differences are correctly non dimensionalized they can be taken to be valid measures of the disturbances.

These considerations suggest the definition of disturbances in terms of overall disturbance parameters, at each time, as the sum of the square of the differences at each grid point, (properly non dimensionalized with respect to a meaningful known quantity related to the experiment, e.g. the imposed boundary conditions). To be more explicit for the study case considered (fluid cell across which a 
temperature difference $\Delta T$ is imposed at the boundaries) if the two values of temperature at grid point " $i$ " $\left(T_{i}\right.$ and $\left.T_{0 i}\right)$ are computed, then an appropriate thermal disturbance parameter $\left(\varepsilon_{T}\right)$ can be defined (for steady state conditions):

$$
\varepsilon_{T}=\frac{1}{s} \sum_{i=1}^{s} \frac{\left(T_{i}-T_{0 i}\right)^{2}}{\Delta T^{2}}
$$

where $\mathrm{s}$ is the number of grid points where $\mathrm{T}$ is computed.

A very suitable definition of a disturbance parameter related to heat fluxes can be taken to be the heat $(\dot{Q})$ transported per unit time from one wall (of surface $\mathrm{S}$ ) at $\mathrm{T}+\Delta \mathrm{T}$ to a parallel one at $\mathrm{T}$ positioned at distance $\mathrm{L}$. In quiescent conditions $(0-\mathrm{g})$ and at steady state a purely diffusive process is established so that $\dot{Q}_{0}=\lambda \frac{\Delta T}{L} S, \lambda$ being the thermal conductivity, $\mathrm{S}$ the walls surface and $\Delta T$ the (constant) temperature difference between the two walls. $Q_{0}$ is an excellent reference parameter that defines the Nusselt number

$$
\left(N_{u}=\dot{Q} / \dot{Q}_{0}\right) \text { where } \dot{Q}=\int_{S} \lambda\left(\frac{\partial T}{\partial n}\right)_{w} d S \text { is }
$$

the heat flow rate (computed at either wall). In the study case therefore the disturbance (TFD) $-(\mathrm{TFD})_{0}$ can be taken to be $\Delta \mathrm{Nu}=\mathrm{Nu}-$ 1 , that gives an overall measure of the distortion of the temperature field prevailing at $0-\mathrm{g}$ induced by convective motion generated by accelerations prevailing in the microgravity environment

\section{Mathematical modeling}

The real microgravity environment for medium/long duration missions (i.e. orbital platforms) consists of a spectrum of accelerations at different frequencies ranging from zero (steady or quasi-steady residual acceleration) to hundreds of Hertz (periodic g-jitter). The velocity, temperature and concentration fields are coupled through the differential balance equations (for mass, momentum, energy and mass species) in presence of a number of g-disturbances.

In this paper the attention is focused on the simulation of typical study cases of Fluid Science (thermal diffusion in a fluid cell, thermodiffusion coefficient measurements experiments).

Following the usual Boussinesq approximation, the physical properties are assumed constant except the density $\rho$ in the driving force appearing in the momentum equation, which is assumed to be a linear function of temperature and concentration :

$$
\rho=\rho_{0}\left[1-\beta_{T}\left(T-T_{0}\right)-\beta_{c}\left(c-c_{0}\right)\right]
$$

The phenomenological equations relating the heat flux and the species mass flux to the thermal and solutal gradients are (De Groot and Mazur $^{3}$ ):

$$
\begin{aligned}
& \underline{j}_{q}=-\lambda \underline{\nabla T}-\rho T c(\partial \mu / \partial c) D^{\prime} \underline{\nabla} c \\
& \underline{j}_{c}=-\rho D \underline{\nabla c}-\rho D_{T} c(1-\mathrm{c}) \underline{\nabla} T
\end{aligned}
$$

where $\lambda$ is the thermal conductivity, $\mu$ the chemical potential of the solute, D the Fickian diffusivity; the cross-coupling terms containing $\mathrm{D}^{\prime}$ and $\mathrm{D}_{\mathrm{T}}$ give rise to the interaction between the thermal and concentration fields even when the mixture is at rest (Dufour and Soret effect). From the Onsager symmetry relation $\mathrm{D}^{\prime}=\mathrm{D}_{\mathrm{T}}$ (Hurle and Jakeman ${ }^{4}$ ).

For $\mathrm{c} \approx \mathrm{c}_{0}<<1$ the species mass flux equation (4) can be written as:

$$
\underline{j}_{c}=-\rho D\left(\underline{\nabla} c+S_{T} c_{0} \underline{\nabla} T\right)
$$

where $\mathrm{S}_{\mathrm{T}}=\mathrm{D}_{\mathrm{T}} / \mathrm{D}$ is the Soret coefficient. The field equations include the continuity, the Navier-Stokes, the energy and species equations for a binary mixture:

$$
\begin{aligned}
& \underline{\nabla} \cdot \underline{V}=0 \\
& \frac{\partial \underline{V}}{\partial \mathrm{t}}+\underline{V} \cdot \underline{\nabla} \underline{V}+\frac{1}{\rho_{0}} \underline{\nabla} p=v \nabla^{2} \underline{V}- \\
& {\left[\beta_{T}\left(T-T_{0}\right)+\beta_{c}\left(c-c_{0}\right)\right]\left[\underline{g}+\sum_{i=1}^{n} b_{i} \omega_{i}^{2} \cos \omega_{i} t \underline{n_{i}}\right]}
\end{aligned}
$$




$$
\begin{aligned}
& \frac{\partial T}{\partial t}+\underline{V} \cdot \underline{\nabla} T=\alpha \nabla^{2} T+\underline{\nabla} \cdot\left[\frac{T c}{C_{p}}(\partial \mu / \partial c) D^{\prime} \underline{\nabla} c\right] \\
& \frac{\partial c}{\partial t}+\underline{V} \cdot \underline{\nabla} c=D\left(\nabla^{2} c+S_{T} c_{0} \nabla^{2} T\right)
\end{aligned}
$$

where $C_{p}$ is the specific heat coefficient, $\alpha$ is the thermal diffusivity, $v$ the kinematic viscosity of the solution; $b_{i}$ and $\omega_{i}$ are respectively the amplitude and the frequency of the i-th oscillatory acceleration component. The initial conditions prescribe zero-velocity and uniform temperature and concentration distribution in the fluid cell. The diffusion process starts when a temperature gradient is applied across the cell.

The boundary conditions are: 1) no slip velocity conditions on the rigid boundaries; 2 ) prescribed temperatures at the hot and cold end walls; 3) adiabatic conditions for the temperature along the lateral wall surfaces; 4) zero mass flux on the boundaries for the concentration.

Equations (6-9) have been nondimensionalized assuming as scaling factors for length, velocity, temperature and mass fraction, respectively $\mathrm{L}, \alpha / \mathrm{L}, \Delta \mathrm{T}=\mathrm{T}_{\mathrm{H}^{-}}$ $\mathrm{T}_{\mathrm{C}}$, the concentration is scaled with the value $\mathrm{S}_{\mathrm{T}} \mathrm{c}_{0} \Delta \mathrm{T}$ corresponding to the rate of separation due to Soret effect in the $0-\mathrm{g}$, "ideal", case (i.e. purely diffusive situation). In this case the concentration is a linear function of the axial coordinate in the ampoule and the values of the scaled variable $c$ reach 0.5 and -0.5 at the cold and hot end, respectively.

The relevant dimensionless parameters are the Rayleigh number, the Prandtl and the Schmidt number defined as:

$$
\operatorname{Rag}=\frac{g \beta_{T} \Delta T L^{3}}{v \alpha} ; \operatorname{Pr}=\frac{v}{\alpha} ; \mathrm{Sc}=\frac{v}{\mathrm{D}}
$$

The relative importance of thermal and solutal buoyancy effects is measured by the parameter $\varepsilon$, defined by:

$$
\varepsilon=\frac{\beta_{c} \Delta c}{\beta_{T} \Delta T}=\frac{\beta_{c}}{\beta_{T}} S_{T} c_{0}
$$

For $\varepsilon>0$ thermal and solutal effects are concurrent, for $\varepsilon<0$ they are counteracting. Under the assumptions of small amplitudes and large frequencies of the oscillatory accelerations, the Gershuni formulation can be applied leading to a closed set of equations for the time-averaged quantities.

The relevant parameter in this case is the vibrational Rayleigh number defined by ${ }^{2}$.

$$
R a_{v}=\frac{\left(b \omega \beta_{T} \Delta T L\right)^{2}}{2 v \alpha}
$$

\section{Reference study case}

The reference study case extensively investigated over the last few years at the University of Naples for the evaluation of the disturbances induced by the ISS microgravity environment ${ }^{5}$ consists in the energy diffusion in a cubical closed fluid cell (like the BDPU test container) (Eq. (9) is not conseidered).

Two walls of the cell (at $\mathrm{x}=0$ and $\mathrm{x}=\mathrm{L}$ ) are maintained at constant temperatures with a typical value of the temperature difference $\Delta \mathrm{T}=50 \mathrm{~K}$; the other boundaries are supposed adiabatic. The size of the cell is $5 \mathrm{~cm}$ and the pure liquid is Silicone oil with a low kinematic viscosity.

To assess the validity and the accuracy of the averaging method the results of the timeaveraged formulation have been compared with the results of the direct numerical simulation of the Navier-Stokes equations with time-dependent accelerations (that give the exact instantaneous time-dependent flow). Figures 1 and 2 show numerical results obtained for the reference study case (Silicone oil $0.65 \mathrm{Cs}$ ) in presence of a g-jitter of amplitude $10 \mathrm{mg}$ at frequency of $3 \mathrm{~Hz}$ and when a residual-g of $1 \mu \mathrm{g}$ is superimposed to the g-jitter (the residual-g and the vibration are assumed in the worst directions i.e. orthogonal to the imposed density gradient). In particular Figures $1 \mathrm{a}$ and $2 \mathrm{a}$ show the contours of the nondimensional y-component of the velocity; Figures $1 b$ and $2 b$ show the vector plots and the temperature distributions. The initial temperature distribution is purely diffusive and the results refer to 600 seconds after the application of the acceleration disturbances. 
Examination of these figures show that the results of the direct and the time-averaged formulations are almost coincident, even

\section{DIRECT FORMULATION}

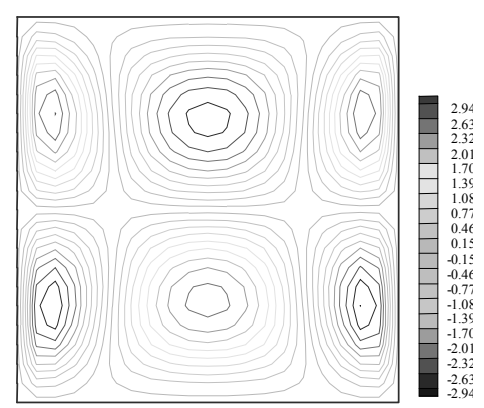

(1a) Non dimensional $\mathrm{Y}$-velocity $(\mathrm{t}=600 \mathrm{~s})$

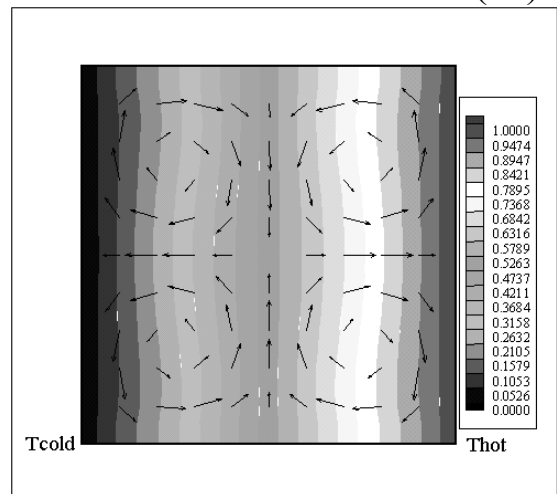

G-jitter

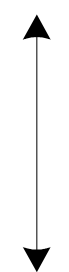

during unsteady phases, for both a single frequency vibration and for the simultaneous application of a residual-g plus vibrations.

\section{TIME-AVERAGED FORMULATION}
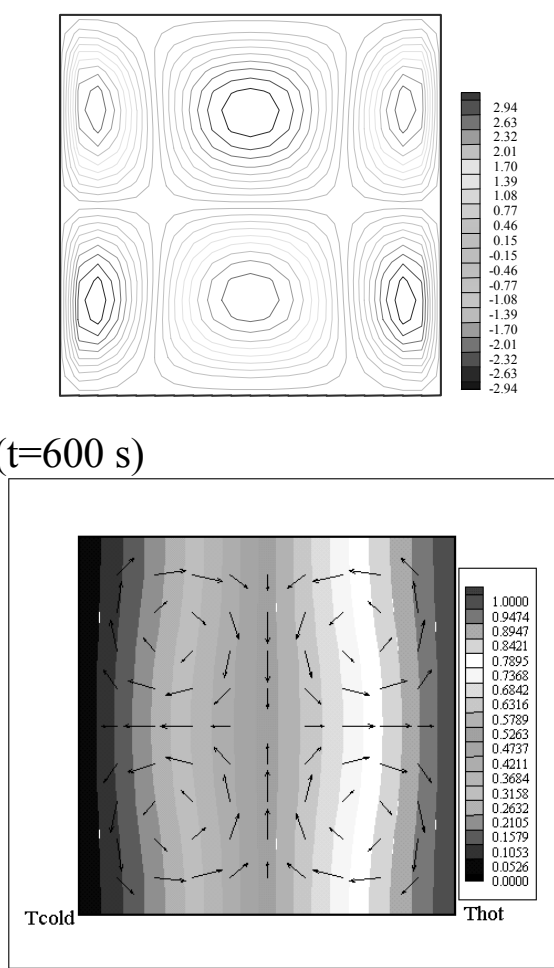

$\mathrm{V}_{\max } / \mathrm{V}_{\alpha}=2.99 \quad$ (1b) Non dimensional Temperature $(\mathrm{t}=600 \mathrm{~s}) \quad \mathrm{V}_{\max } / \mathrm{V}_{\alpha}=2.97$
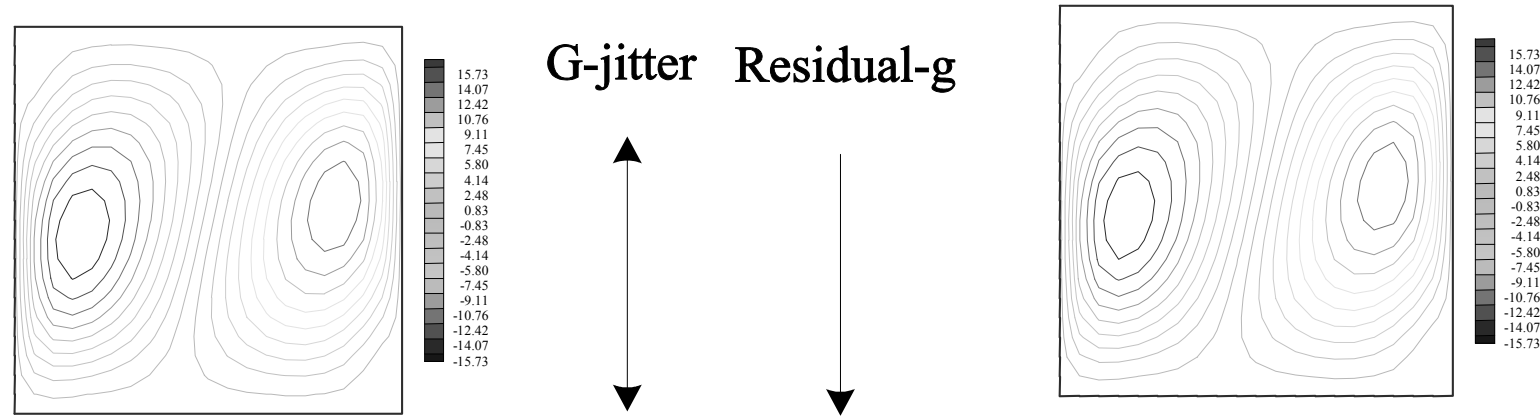

(2a) Non dimensional Y-velocity $(\mathrm{t}=600 \mathrm{~s})$

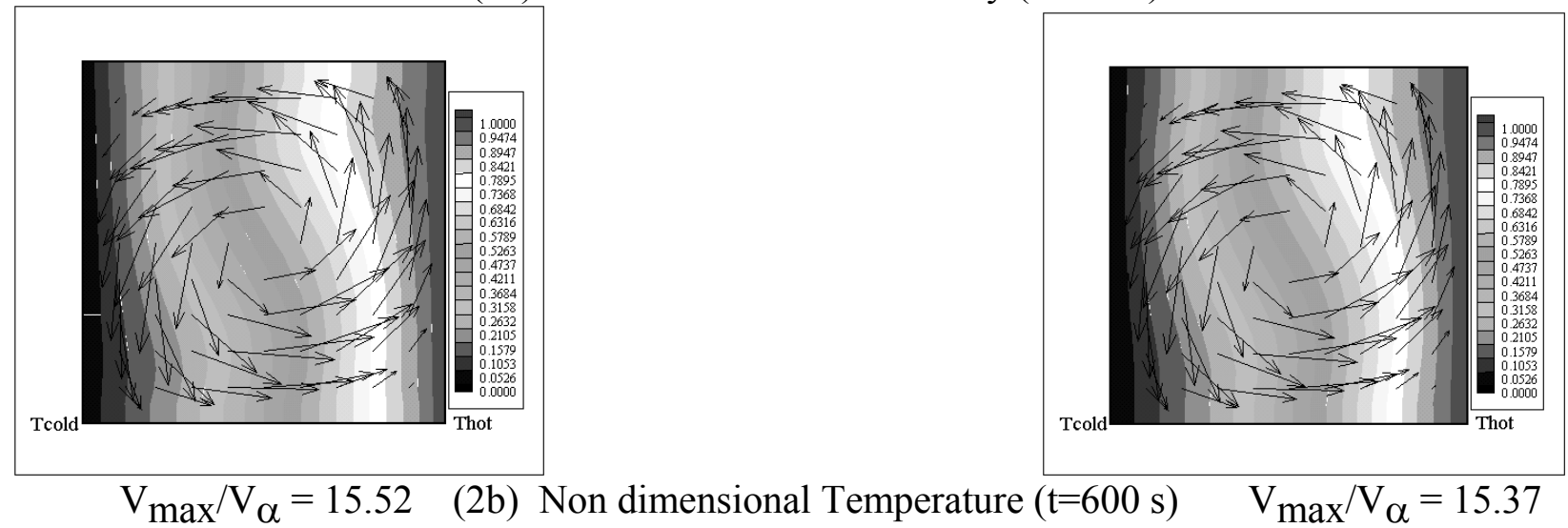

Figs 1 and 2: comparison between direct and time-averaged formulations for g-jitter only (1) and simultaneous g-jitter and residual-g (2) 
5. Influence of residual-g and g-jitter at different ISS locations

The initial time-averaged formulation for single-frequency sinusoidal disturbances has been extended to the real situation characterized by a spectrum of frequencies simultaneously acting on the experiment cell (that represents the real ISS environment). As shown theoretically and confirmed by extensive numerical analysis 5 the timeaveraged disturbances can be computed with less computation time by solving the timeaveraged form of the field equations, containing all the g-jitter terms (like those that identify the MGE of the ISS) grouped in a single parameter, by introducing a suitable defined equivalent vibrational Rayleigh number given by the sum of the vibrational Rayleigh numbers corresponding to the different sinusoidal functions of the spectrum:

$$
\mathrm{Ra}_{\mathrm{V}}{ }^{(\mathrm{e})}=\sum_{\mathrm{i}=1}^{\mathrm{m}} \mathrm{Ra}_{\mathrm{Vi}} ; \mathrm{Ra}_{\mathrm{Vi}}=\frac{\left(\mathrm{b}_{\mathrm{i}} \omega_{\mathrm{i}} \beta_{\mathrm{T}} \Delta \mathrm{TL}\right)^{2}}{2 v \alpha}
$$

This means that the overall effect of many simultaneously acting vibrations can be easily numerically computed assigning as input to the CFD code an "equivalent" single frequency g-jitter.

Numerical simulations have been carried out to evaluate the residual-g and g-jitter effects on the same experimental cell located inside different modules of the ISS.

The accelerations given as input to the CFD code correspond to the recent NIRA 99 predictions for the US lab and for the ESACOF. Due to the large extension of the ISS and to the different vibrations induced by crew activities on the US Lab and on the ESA-COF module, the Space Station can be seen as an ensemble of microgravity platforms with substantial differences of the microgravity environments encountered by the experimental facility. In particular the recent NASA models predict a value of the residual-g of the order of $0.5 \mu \mathrm{g}$ for the US $\mathrm{Lab}$ and of about $1.6 \mu \mathrm{g}$ for the ESA-COF.

On the other hand the g-jitter disturbances are expected to be larger in the US Lab than in the European COF (see Fig. 3).
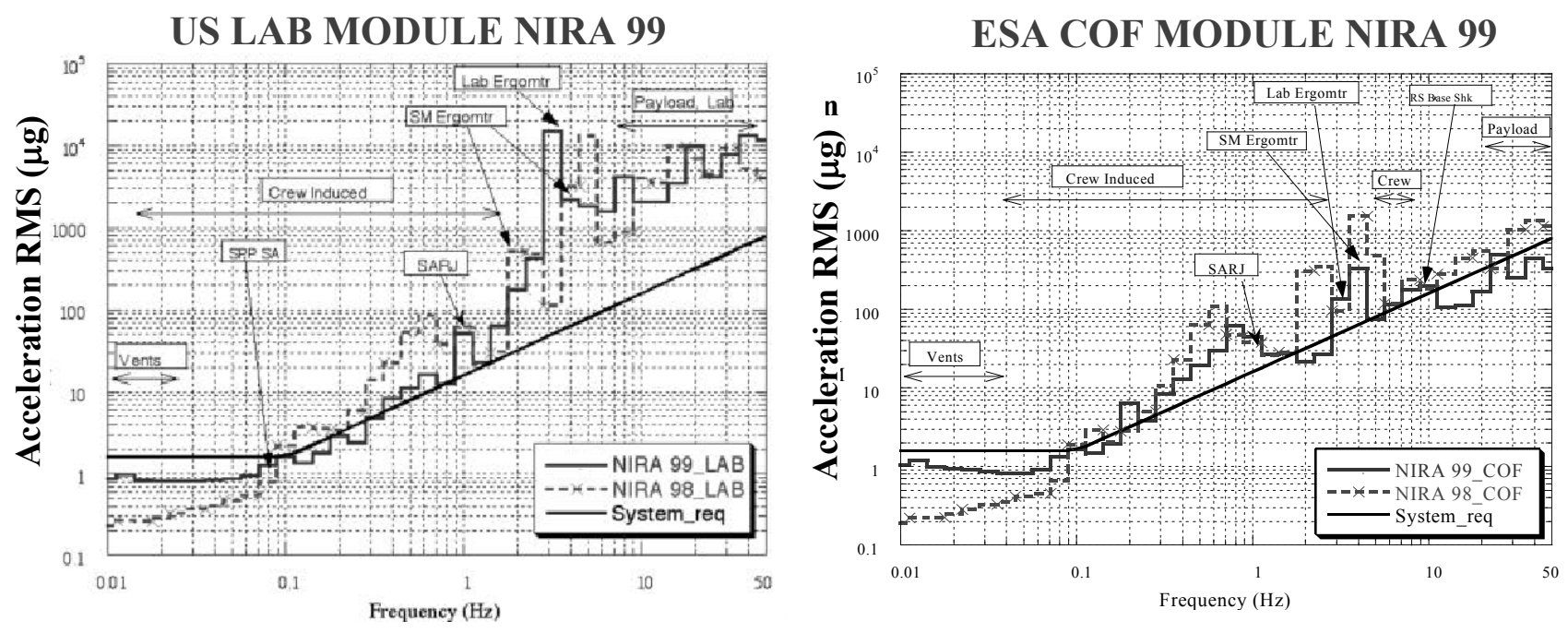

Figs.3: NIRA 99 predictions for the US lab and for the ESA-COF

Is is essential to recognize that the main difference between the US Lab and ESA COF is mainly due to the oscillation induced by the
Lab Ergometer that is planned to be positioned inside the Us Lab. In fact the rms of the amplitudes, at a frequency close to $3 \mathrm{~Hz}$, induced by this equipment (see Fig.3), is respectively about $15000 \mu \mathrm{g}$ for the US Lab 
and about $150 \mu \mathrm{g}$ for COF. Provisions are been taken to reduce these accelerations in an updated, non official DAC-8. For this version Lab ergometer vibrations appear to be one order of magnitude less (about $1000 \mu \mathrm{g}$ for US Lab).

The microgravity community sees this Lab Ergometer as a "drifting mine" because, apparently, no decision is being taken yet where it will be eventually located.

Figures 4, 5, 6 summarize the numerical results for the reference study case. Fig. 4 compares the computed streamlines, vector plots and temperature distributions for the US-Lab and the ESA-COF environments, when only g-jitter is present (i.e. in the unrealistical situation that the residual-g is zero and the cell is subjected only to the vibrations predicted for the US Lab or for the ESA-COF).
Since the predicted vibrations are larger for the US-Lab module (see again Fig. 3) the convective disturbances and the temperature distortions are larger for the US Lab. Figure 5 shows the numerical results obtained in the other extreme situation that only residual-g is present $(0.5 \mu \mathrm{g}$ for the US Lab and $1.6 \mu \mathrm{g}$ for the ESA-COF) without any g-jitter. The situation is reversed, i.e. the convective disturbances are large in the ESA-COF, due to the larger value of the residual gravity.

Fig. 6 shows the results obtained assuming that both residual-g and g-jitter are present. In this case the overall convective disturbances (measured e.g. by the Nusselt number) are comparable in value, but in the US Lab the major cause of disturbances is the g-jitter, on the contrary in the ESA-COF it is the residual-g.
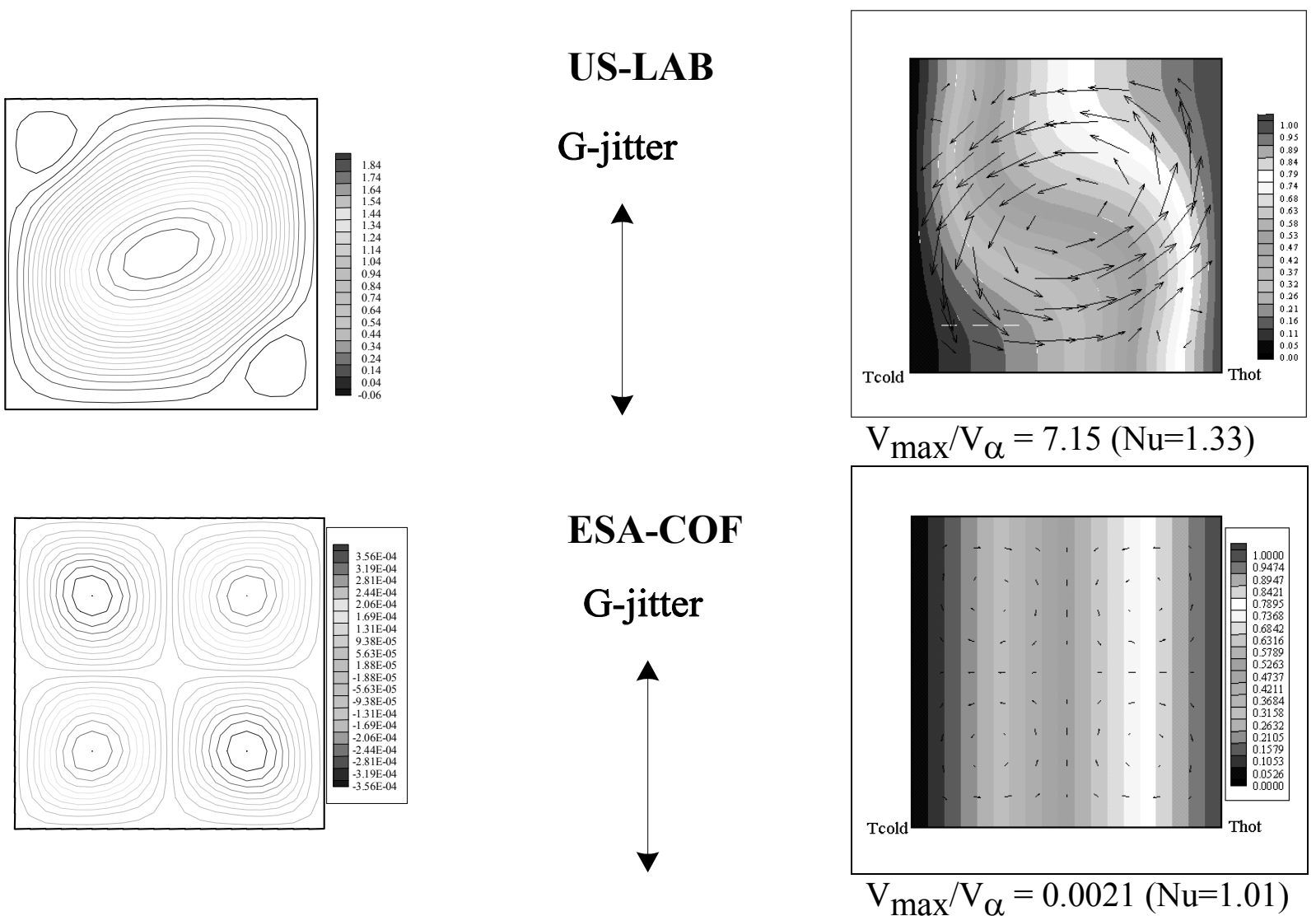

Figs.4: Computed streamlines, vector plots and temperature distributions for the US-Lab and the ESA-COF, when only g-jitter is present (time averaged code, steady state) 

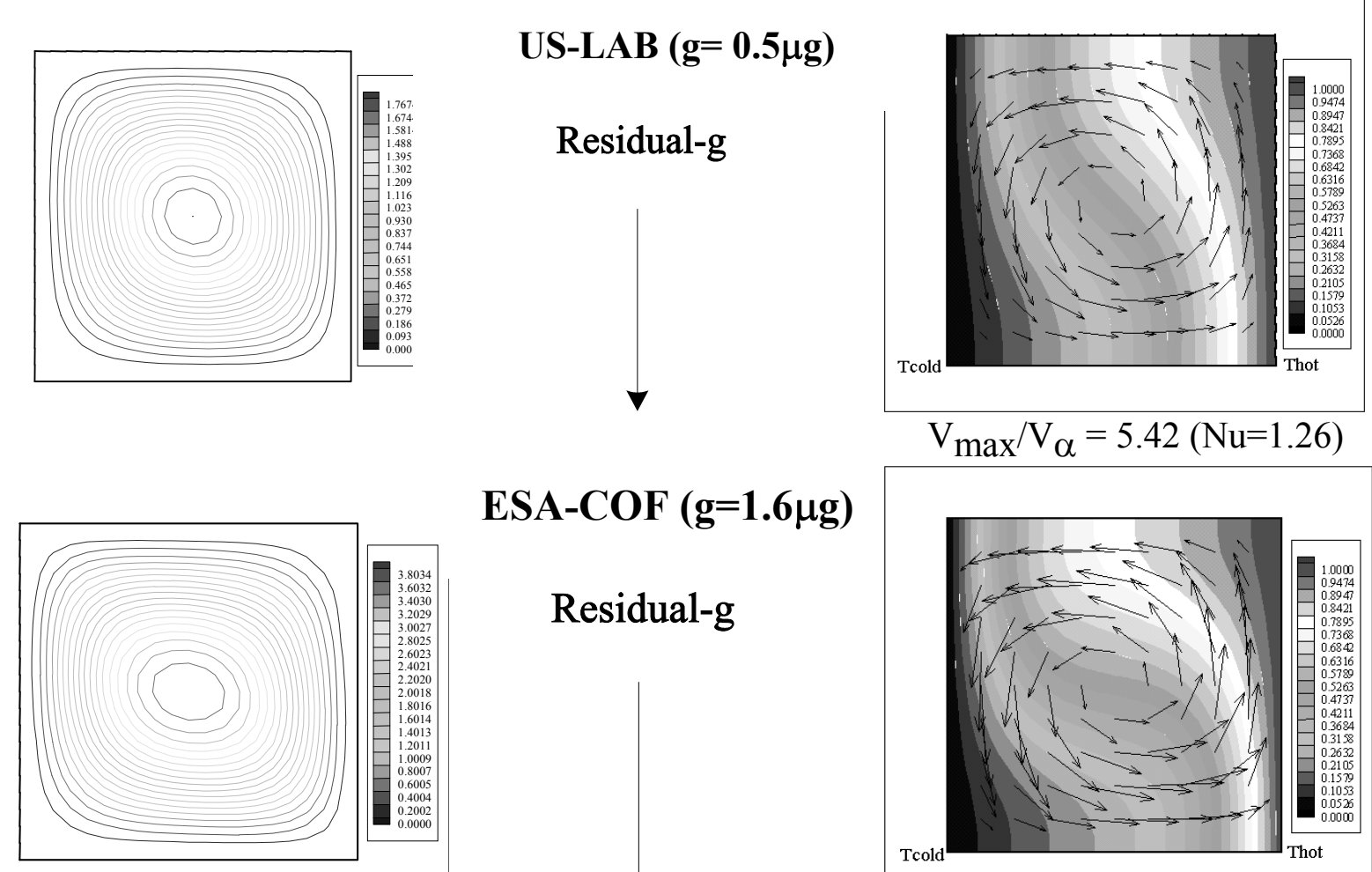

\section{ESA-COF $(g=1.6 \mu g)$}

\section{Residual-g}
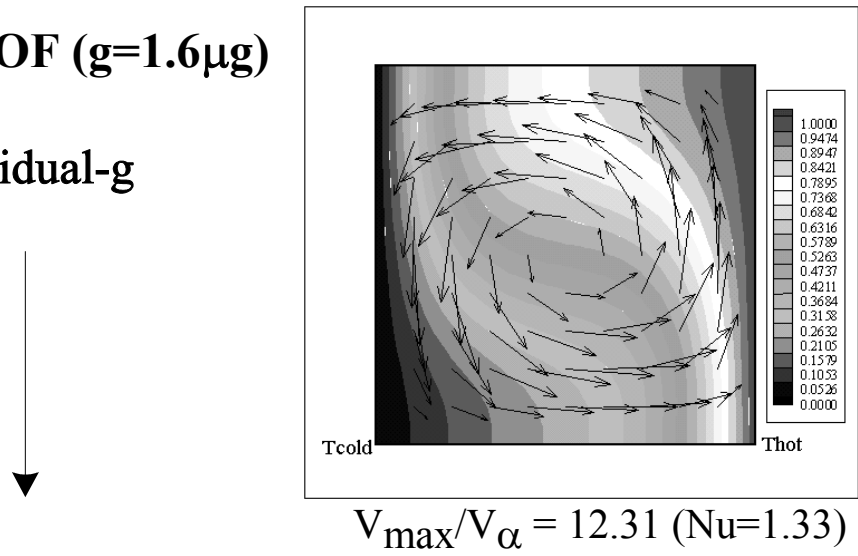

Figs.5: computed streamlines, vector plots and temperature distributions for the US-Lab and the ESA-COF, when only residual-g is present (steady state)
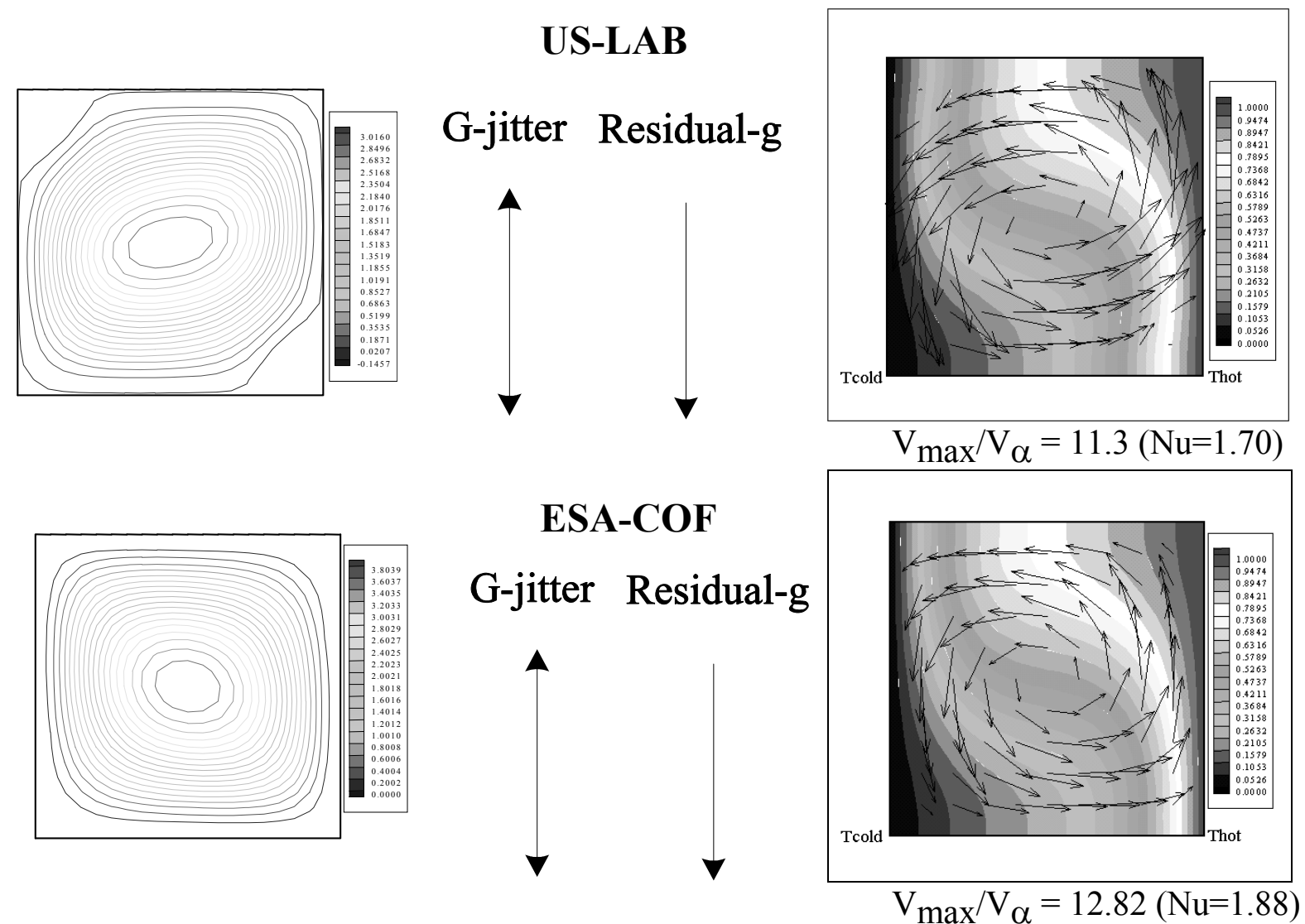

Figs.6: computed streamlines, vector plots and temperature distributions for the US-Lab and the ESA-COF, when residual-g and g-jitter are both present (time averaged code, steady state). 


\section{$\underline{6 . \text { Influence of cell orientation }}$}

Orienting the experiment cell with the density gradient along the residual-g or along the gjitter can help mitigating the convective disturbances ${ }^{6}$.

Numerical simulations have been carried out to evaluate the influence of the cell orientation with respect to the residual-g and g-jitter vectors in the US Lab and in the European COF modules.

Figs. 7 show the different thermofluidynamic fields obtained changing the orientation of the residual-g and g-jitter vectors.

The TFD distortions in the US Lab are minimized when the cell is oriented with the density gradient parallel to the g-jitter direction (main source of disturbances) ; in this case the stabilizing effect of the g-jitter can also be beneficial in minimizing the destabilizing effect of a residual-g parallel to the temperature gradient (i.e. like a cell heated from below on Earth).

\section{US-LAB}

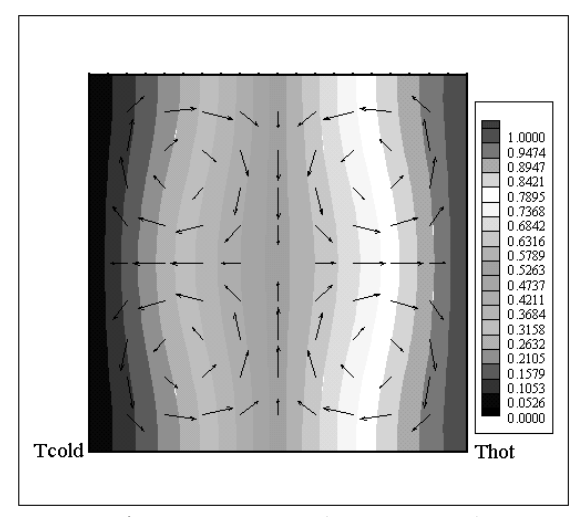

$\mathrm{V}_{\max } / \mathrm{V}_{\alpha}=2.99(\mathrm{Nu}=1.04)$

\section{US-LAB}

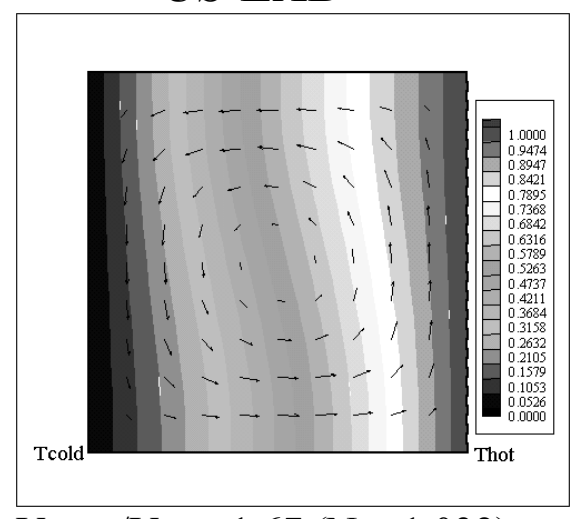

Stabilizing residual-g

Orthogonal g-jitter

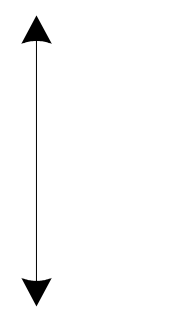

Orthogonal residual-g

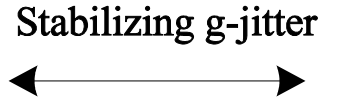

ESA-COF

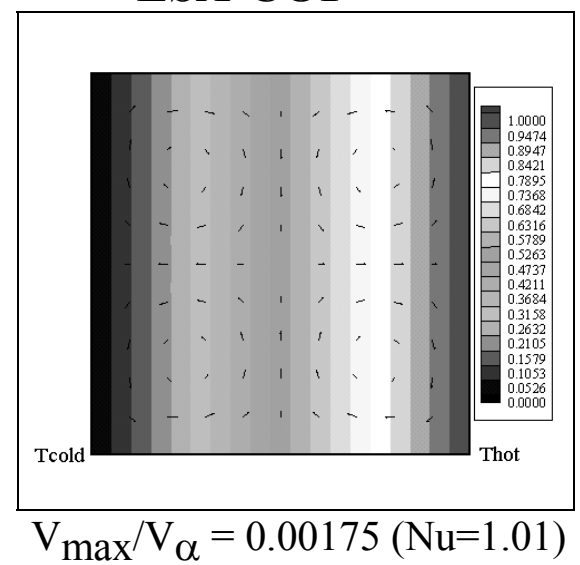

ESA-COF

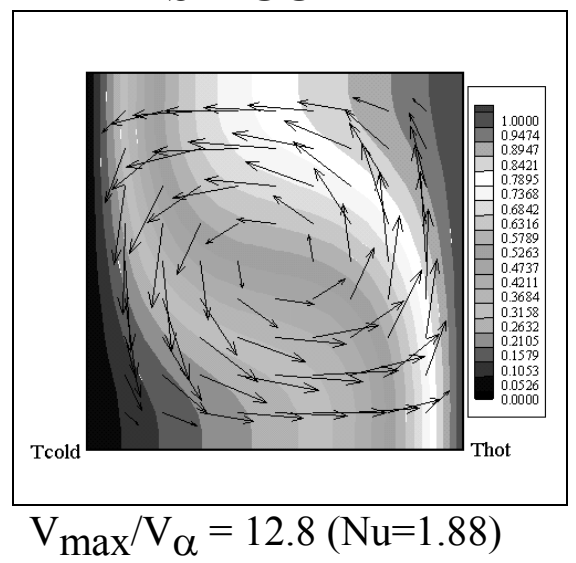

Figs.7: Vector plots and temperature distributions for the US-Lab and the ESA-COF, when residual-g and g-jitter have different orientation (time averaged code, steady state).

In the European COF the convective disturbances are minimized only when the residual-g vector is antiparallel to the temperature gradient (i.e. stabilizing), even in the presence of an orthogonal g-jitter.

In conclusion it is possible to find the "best" cell orientation in both the Modules. Figure 8 and Table 1 summarize the computed Nusselt numbers for different cell orientations. It appears that orientation is more efficient than Isolation Mounts to minimize convective disturbances.

However it should be pointed out that, when residual-g is negligible, orientation works only if the dominant g-jitter direction is known. 


\section{Nusselt Number}

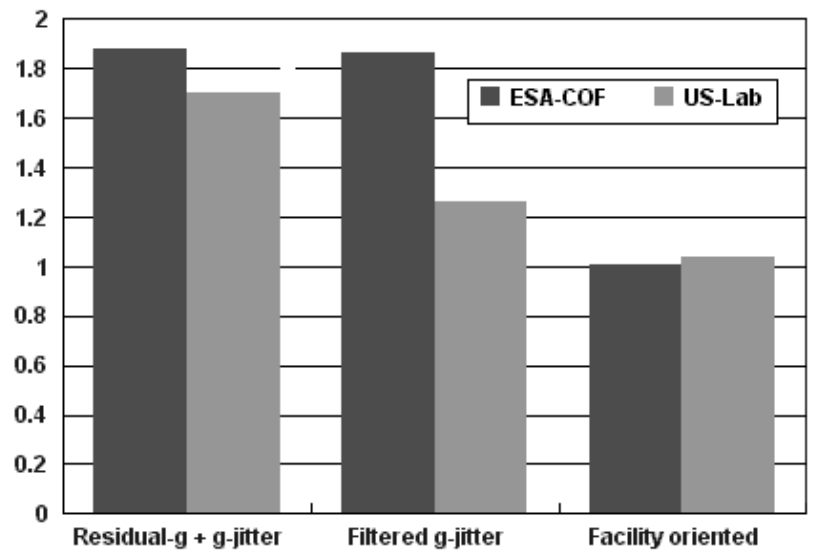

\begin{tabular}{|c|c|c|c|c|}
\hline & $\alpha_{G}$ & $\alpha_{J}$ & $\begin{array}{c}\text { Nusselt Number } \\
\text { (U S Lab) } \\
\text { Residual-g=05 } \\
\text { G-Jike : NIRA } 9915 \text { freq. }\end{array}$ & $\begin{array}{l}\text { Nusselt Number } \\
\text { (E SA C OF) } \\
\text { Residual-g }=1.6 \text { kg } \\
\text { G-Fiter : NIRA } 9915 \text { freq. }\end{array}$ \\
\hline Residual-G & -90 & $>$ & 1.26 & 1.88 \\
\hline G-Jitter & $<$ & -90 & 1.33 & 1.00 \\
\hline Residual-G+Jitter & -90 & -90 & 1.70 & 1.88 \\
\hline Residual-G+Jitter & 180 & -90 & 1.04 & 1.00 \\
\hline Residual-G & $\mathbf{0}$ & $<$ & 1.00 & 1.76 \\
\hline Residual-G+Jitter & $\mathbf{0}$ & -90 & 1.61 & 1.76 \\
\hline G+Jitter & $<$ & 180 & 1.00 & 1.00 \\
\hline Residual-G+Jitter & -90 & 180 & 1.03 & 1.88 \\
\hline Residual-G+Jitter & 180 & 180 & 1.00 & 1.00 \\
\hline Residual-G+Jitter & $\mathbf{0}$ & 180 & 1.00 & 1.76 \\
\hline
\end{tabular}

Fig. 8 and Table 1: Computed Nusselt numbers for different cell orientations.

\section{Thermodiffusion in metal alloys}

We consider next a typical experiment for the measurement of the thermodiffusion (Soret) coefficient in a typical metal alloy.

The case differs from the previous study case mainly because the density gradient is due to both temperature and concentration gradients. The experiment procedure consists in applying a large temperature difference across a cell containing the melt of a pure metal with an initial uniform concentration of a second component. A heat transfer by diffusion takes place due to both the temperature and concentration gradients [Dufour effect, see eq. (3)]. At the same time diffusion of the secondary component (Soret effect) takes place according to eq. (4) that creates a concentration gradient..

Initially $\underline{\nabla c}=\underline{0} \quad$ and $\quad \underline{\nabla} \neq \neq 0$ and the component starts diffusing toward the cold wall (for positive Soret coefficient) and creating a concentration gradient that, along the cell axis " $x$ ", in a purely diffusive (quiescent) one dimensional process evolves in time and in $\mathrm{x}$ together with the temperature difference

$$
\underline{\nabla} T(x, t) \quad \underline{\nabla c}(x, t)
$$

Establishment of purely one-dimensional heat and species transport calls for adiabatic and impermeable lateral walls:

$$
\left(\frac{\partial T}{\partial y}\right)_{y=0}=\left(\frac{\partial T}{\partial y}\right)_{y=H}=0
$$

$\left(\frac{\partial c}{\partial y}\right)_{y=0}=\left(\frac{\partial c}{\partial y}\right)_{y=H}=0$

$\mathrm{H}$ being the distance of the lateral walls.

Typically for metal alloys, for which the Lewis number $\left(\frac{\alpha}{D}\right)$ is very large $\left(10^{4}\right.$, for the examined case) one can assume that the thermal evolution takes place much faster than the diffusion process so that imposing a temperature difference $\Delta \mathrm{T}$ across the cell (of length L) a constant temperature gradient is "immediately" established in times of the order of $\frac{L^{2}}{\alpha} \cong 0\left(10^{2}[s]\right)$; during this time the species diffusion process has hardly initiated (the characteristic time for the species diffusion is about $10^{4}$ times larger than that for heat diffusion). Furthermore the conditions are such that the Dufour effect (i.e. the heat transport associated with species diffusion) is negligible with respect to the direct heat diffusion since

$$
\lambda \underline{\nabla T}>>\rho T c(\partial \mu / \partial c) D^{\prime} \underline{\nabla c}
$$

In particular the order of magnitude of the ratio of these two fluxes is about $10^{3}$

From eq. (8) one may assume that a constant temperature gradient is established along the cell after times of the order of $10^{2}[\mathrm{~s}]$; the conservation of energy equation, if no convection is present $(\underline{\mathrm{V}}=0)$ :

$$
\frac{\partial T}{\partial t}=\alpha \frac{\partial^{2} T}{\partial x^{2}}
$$


at a steady state condition $\left(\frac{\partial}{\partial t} \equiv 0\right)$ yields

$\frac{\partial^{2} T}{\partial x^{2}} \equiv 0 ; \quad \frac{\partial T}{\partial x}=$ const $=\frac{\Delta T}{L}$

Eq. (4), after an initial thermal transient, reads:

$\underline{j}_{c}=-\rho D \underline{\nabla}_{C}-\rho D_{T} c_{0} \frac{\Delta T}{L} i$

Due to the impermeable boundary conditions at the end walls $(x=0, L)$ :

$j_{c}=0 \Rightarrow\left(\frac{\partial c}{\partial x}\right)_{0, L}=-S_{T} c_{0}\left(\frac{\partial T}{\partial x}\right)_{0, L}$

After an initial transient a steady state is achieved also for the one dimensional concentration distribution $\left(\frac{\partial c}{\partial t} \equiv 0\right)$ and, in absence of any convection, the separation coefficient $\left(\mathrm{S}_{\mathrm{T}}\right)$ can be simply related to the difference in concentration $(\Delta c)$ at steady state at the two ends of the cell:

$$
S_{T}=-\frac{1}{c_{0}} \frac{\Delta c}{\Delta T}
$$

In presence of convection the field is twodimensional; if one tries to use the previous formula, $\Delta \mathrm{c}$ should be computed as the difference between the average concentration at the two cell ends:

$$
\Delta c=\frac{1}{H}\left[\int_{0}^{H} c(0, y) d y-\int_{0}^{H} c(L, y) d y\right]
$$

this value tends to be smaller because of the mixing induced by velocities and the apparent measured separation coefficient is smaller than the real one.

On the contrary when trying to measure an isothermal diffusion coefficient starting from two liquids at different concentrations, brought in contact and free to diffuse one into the other then any convective effects is responsible for an overestimation (the apparent diffusion coefficient is larger than the real one). For the present computations a cell with length $\mathrm{L}=4 \mathrm{~cm}$ and height $\mathrm{H}=1 \mathrm{~cm}$ is considered.

The values of the thermophysical parameters have been assigned considering a typical experiment with metal alloys ${ }^{7}\left(v=10^{-7} \mathrm{~m}^{2} / \mathrm{s}\right.$, $\mathrm{D}=10^{-9} \mathrm{~m}^{2} / \mathrm{s}, \quad \alpha=10^{-5} \mathrm{~m}^{2} / \mathrm{s}, \quad \beta_{\mathrm{T}}=10^{-4} \mathrm{~K}^{-1}$, $\left.\mathrm{S}_{\mathrm{T}}=10^{-3} \mathrm{~K}^{-1}, \Delta \mathrm{T} / \mathrm{L}=10^{2} \mathrm{~K} / \mathrm{cm}, \varepsilon=0.2\right)$.
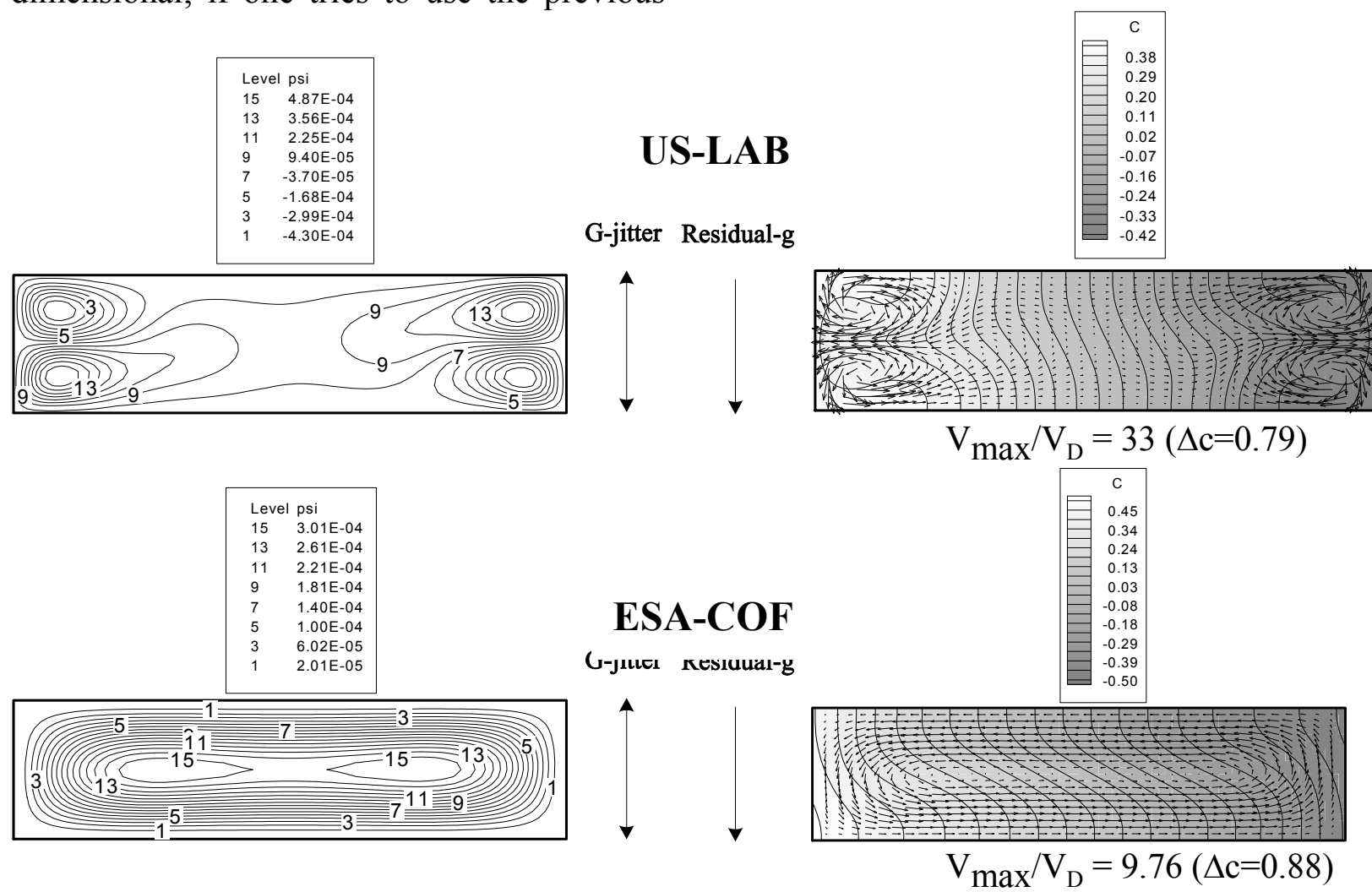

Fig. 9: Streamlines, vector plots and concentration distribution for residual-g and g-jitter both orthogonal to the density gradient 

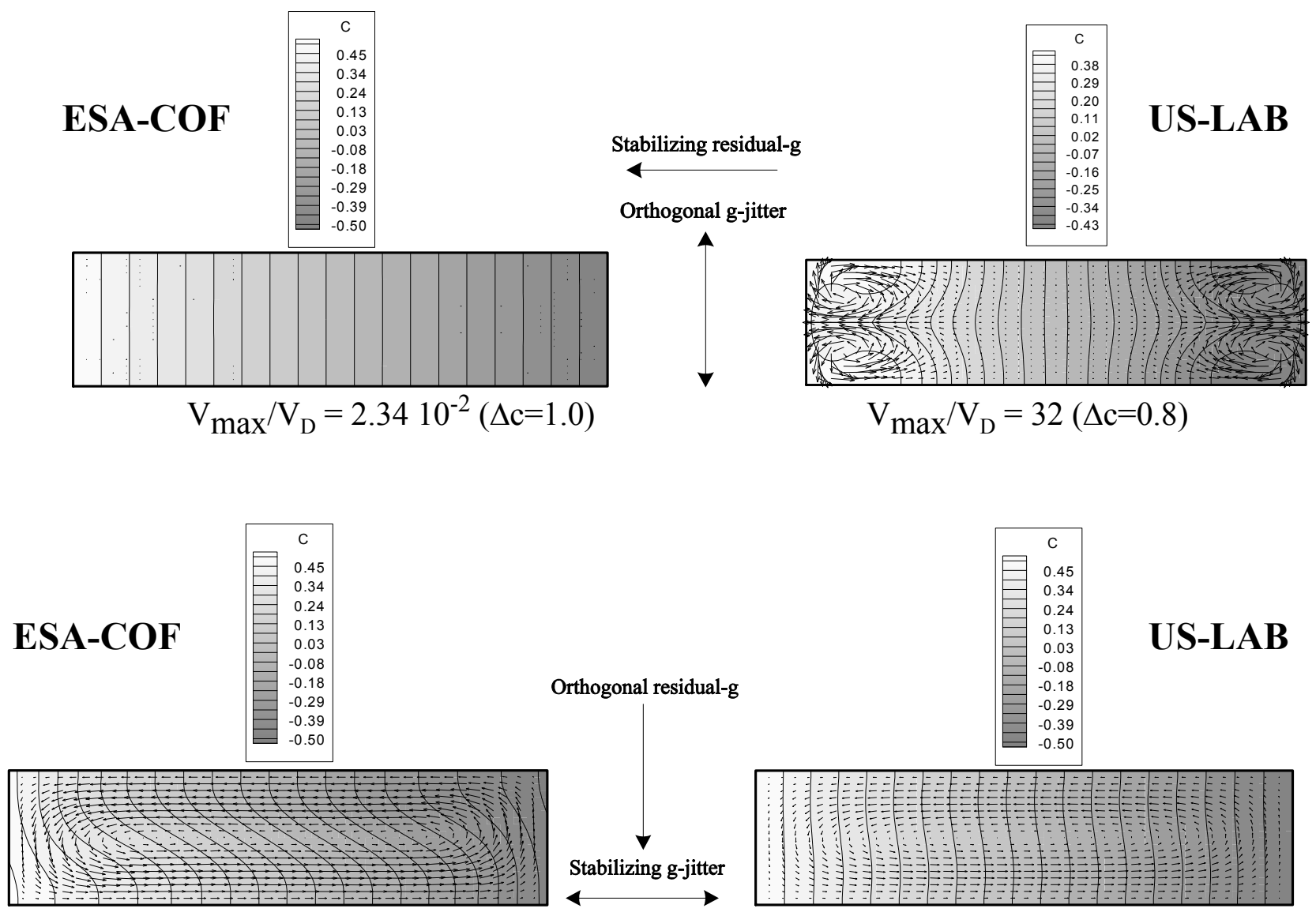

$\mathrm{V}_{\max } / \mathrm{V}_{\mathrm{D}}=9.6(\Delta \mathrm{c}=0.88)$

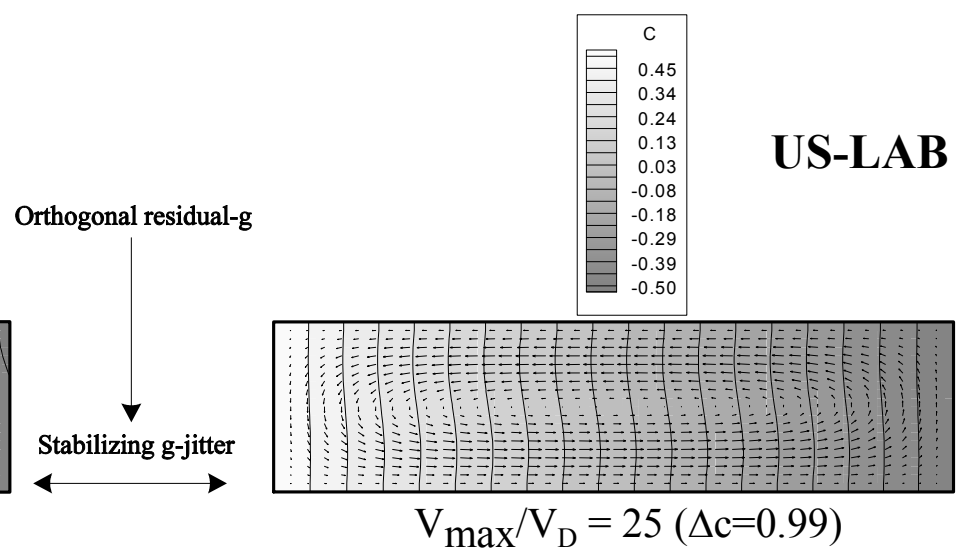

Fig. 10: Vector plots and concentration distributions for residual-g and g-jitter at different orientations

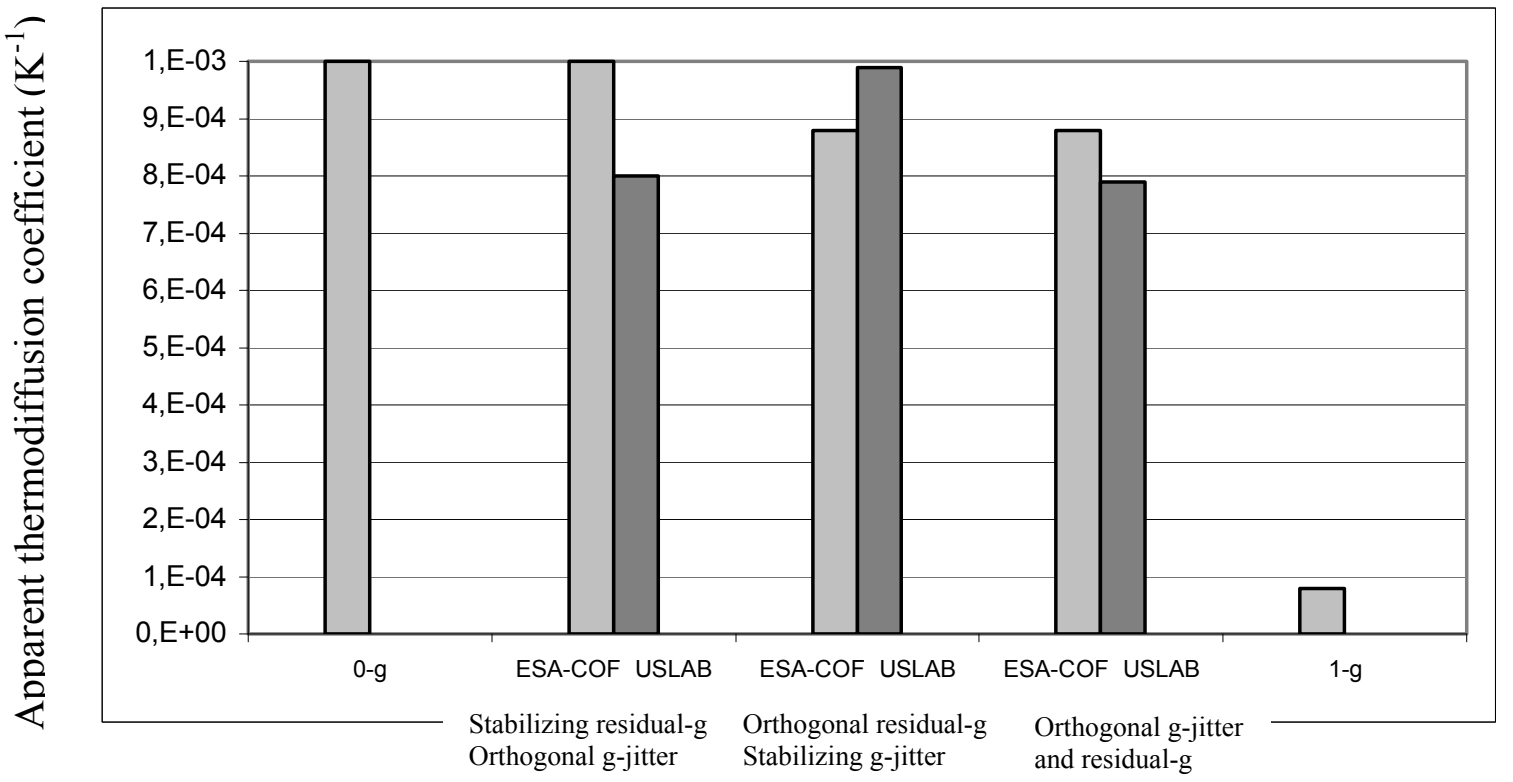

Fig. 11: Apparently measured thermodiffusion coefficient 
Preliminary numerical simulations have been carried out to validate the code. As for the reference study case also for this problem the results of the direct and time-averaged formulations were almost the same, with differences less than $1 \%$ between the two codes. The results obtained with the Gershuni formulation, assuming the microgravity environments in the UsLab and in the ESACOF shown in Figs. 3, are shown in Figs. 9 and 10 and summarized in Fig. 11, where the values of the thermodiffusion coefficient evaluated by the concentration difference $\Delta \mathrm{c}$ are reported. Examinations of Figs. 9. 10 and 11 shows that this experiment is highly microgravity relevant (no separation occurs in 1-g due to the very strong convection induced on Earth) and that the apparently measured thermodiffusion coefficient, in the worst situation that residual-g and g-jitter are both orthogonal to the density gradient, is about $30 \%$ smaller than the real one. The cell orientation can be beneficial to reduce convective disturbances. In the US Lab (large g-jitter and small residual-g) the cell should be oriented with the density gradient axis parallel to the axis of the main source of vibration. In the COF (rather large residual-g and small g-jitter) the cell should be oriented with the density gradient axis parallel to the residual-g.

\section{$\underline{\text { 8. Summary and conclusions }}$}

A numerical tool has been prepared to quickly predict the overall g-jitter disturbances for the typical microgravity environment prevailing on the ISS. The code has been employed to simulate a simple reference study case and applied to the problem of thermodiffusion in a metal alloy.

Numerical simulations have been performed for cells located at different locations of the ISS (US Lab and ESA-COF) and at different orientations with respect to the residual-g ang g-jitter vectors.

The results show that Isolation Mounts that strongly reduce "high" frequency g-jitters are probably justified only in ISS locations (like the US Lab) where very low values of the residual-g prevail and relatively large g-jitter are predicted (especially g-jitter induced by the ergometer present in the US Lab). On the contrary, if residual-g effects prevail over gjitter effects (like in the COF module), Isolation Mounts are not justified.

In presence of both residual-g and g-jitter the cell orientation can be beneficial to reduce convective disturbances.

Microgravity experiments will be performed during the UF\#3 flight (2003), using an orientable drawer, to check the above conclusions for a number of Fluid Physics experiments ${ }^{8}$.

\section{Acknowledgements}

This work was partially supported by the European Space Agency (ESA) and by the Italian Space Agency (ASI).

\section{References}

[1] Savino R, Monti R., "Fluid-Dynamic Experiment Sensitivity to accelerations prevailing on microgravity platforms", in Physics of Fluids in Microgravity, Gordon and Breach (2000).

[2] Gershuni G.Z., Lyubimov D.V., "Thermal Vibrational Convection", Wiley\&Sons (1998)

[3] DeGroot S.R., Mazur P., "Non Equilibrium Thermodynamics", North Holland (1962).

[4] Hurle D.T.J., Jakeman E., "Soret-driven thermosolutal convection", J.Fluid Mechanics 47, pp.667-687(1971).

[5] Savino R., "Order of magnitude analysis of $\mathrm{g}$-jitter induced disturbances in fluiddynamic microgravity experimentation", Microgravity Quarterly 7, N. 4, pp. 141-154 .

[6] Savino R., Monti R., "Improving diffusion controlled microgravity experiments by facility orientation". Journal of Aerospace Engineering 212, Part G, pp. 415-426 (1999).

[7] Garandet J.P. et al., "The effect of natural convection on the measurement of mass transport coefficients in the liquid state", in Materials and Fluids under Low Gravity, pp.253-259, Springer Verlag, Berlin (1995)

[8] Savino R., " Influence of residual-g and gjitter on the measurement of thermophysical properties in microgravity", $33^{\text {rd }}$ COSPAR Scientific Assembly, 16-23 July 2000, Warsaw, Poland 